Revista Perspectivas Online: Biológicas \& Saúde Fevereiro de 2020, Vol. 10, n ${ }^{\circ} 32$, p.16-22

ISSN: 2236-8868 (Online)

DOI: $10.25242 / 8868103220201954$

\title{
EFEITO AGUDO DA REALIZAÇÃO DO TRÍCEPS NA POLIA NO ESTRESSE FUNCIONAL NO SUPINO RETO
}

\author{
Gabriel Monteiro de Almeida ${ }^{*}$; Igor da Silva Almeida ${ }^{1}$ \& Anderson Pontes Morales ${ }^{1}$
}

ALMEIDA, G.M.; ALMEIDA, I.S.; MORALES, A.P. Efeito agudo da realização do tríceps na polia no estresse funcional no supino reto. Perspectivas Online: Biológicas \& Saúde, v.10, n.32, p.16-22, 2020.

\section{RESUMO}

O objetivo deste estudo foi verificar a magnitude da realização do tríceps na polia no estresse funcional no supino reto. A amostra foi composta por 10 indivíduos, do sexo masculino, com idade de 24,9 $\pm 3,17$ anos, massa corporal de $78,9 \pm 11,48 \mathrm{~kg}$ e estatura de $1,79 \pm 0,05 \mathrm{~cm}$. Os procedimentos consistiram na realização do exercício supino reto. Em outro momento a realização do supino reto imediatamente após a realização do tríceps na polia. Os resultados revelaram que o estresse funcional do tríceps braquial provocado pela execução do tríceps na polia subsequente à execução do supino reto foi de $10.06 \%$ na $1^{\mathrm{a}}$ série $(\mathrm{p}<0,05)$, no total de 3 séries. Conclui-se que a execução do exercício de tríceps na polia precedido ao exercício de supino resultou no estresse funcional somente na $1^{\mathrm{a}}$ série e, que a presença da fadiga do músculo peitoral apresentada nas séries subsequentes não impactou no aspecto funcional do exercício de supino.

Palavras-chave: Desempenho; Fadiga Muscular; Força Muscular.

\begin{abstract}
The objective of this study was to verify the magnitude of the triceps performance in the pulley in functional stress in the bench press. The sample consisted of 10 individuals, male, aged 24.9 \pm 3.17 years, body mass of $78.9 \pm 11.48 \mathrm{~kg}$ and height of $1.79 \pm 0.05 \mathrm{~cm}$. The procedures consisted of performing the bench press exercise. At another time, the bench press is performed immediately after the triceps on the pulley. The results revealed that the functional stress of the triceps brachii Keywords: Performance; Muscle fatigue; Strength.

caused by the execution of the triceps in the pulley after the execution of the bench press was $10.06 \%$ in the $1^{\text {st }}$ series of execution $(\mathrm{p}<0.05)$, in the total of 3 series. It is concluded that the performance of the triceps exercise on the pulley preceded by the bench press exercise resulted in functional stress only in the $1^{\text {st }}$ series and that the presence of chest muscle fatigue presented in the subsequent series did not impact the functional aspect of the bench press exercise.

\footnotetext{
${ }^{1}$ Institutos Superiores de Ensino do CENSA - ISECENSA - Laboratório de Biociências da Motricidade Humana

(*) e-mail: gabrielmonteiro280@yahoo.com.br

Data de recebimento: 27/01/2020. Aceito para publicação: 18/02/2020.
} LABIMH/ISECENSA - Rua Salvador Correa, 139, Centro, Campos dos Goytacazes, RJ, CEP: 28035-310, Brasil;
\end{abstract}




\section{INTRODUÇÃOO}

Atualmente existem diferentes métodos de treinamento que são utilizados para desenvolver determinados tipos de ganhos para indivíduos que desejam obter aumento no desempenho de força, potência (JORGE et al., 2012) e hipertrofia (ORNELAS et al., 2017), dentro das academias de musculação. $\mathrm{O}$ treinamento resistido (TR) é a prática de exercícios físicos em que resistências externas são utilizadas para gerar um estímulo e melhorar a capacidade funcional dos músculos (KRAEMER et al., 2001), possibilitando diferentes resultados a quem o pratica.

O TR é realizado em relação a diversas variáveis, sendo elas, a intensidade de cargas, o número de repetições e séries realizadas, o intervalo entre as séries, a ordem de exercícios e a velocidade de execução dos exercícios. Essas diferentes formas de treinamento e a manipulação dessas variáveis têm resultado em efeitos diferentes nas formas e aprimoramento da força, potência, hipertrofia e resistência muscular (KRAEMER \& RATAMESS, 2000).

Uma das adaptações ao TR é o aumento no desempenho nos níveis de força, contudo, existem alguns aspectos que influenciam diretamente o desempenho e, conseqüentemente, as adaptações agudas ao TR (ACSM, 2009). Dentre eles, destacamos os mecanismos de fadiga central e/ou periférica (ASMUSSEN, 1979; GUYTON \& HALL, 2006). Estudos de AUGUSTSSON et al (2003) e de GENTIL et al (2007) sinalizam que um dos efeitos da fadiga (central e/ou periférica) são as alterações funcionais e eletromiográficas dos músculos, durante a execução do método de pré-exaustão.

Modelos mecânicos (PAOLI, MARCOLIN \& PETRONE, 2009), técnicas eletromiográficas (ESCAMILLA et al., 2001; ESCAMILLA et al., 2009), ressonância magnética (HÄKKINEN et al., 2001) e tomografia computadorizada (TESCH, 1999) são utilizados para verificar as adaptações crônicas ou agudas dos músculos no exercício de força. Não há um consenso na literatura para a conceituação das funções musculares, como também para a relevância da participação do tríceps braquial (TB) nos exercícios multiarticulares para o treinamento prioritário do peitoral, em específico, no supino reto (SR). Com isso, profissionais que fazem uso do TR para suas ações podem estar provocando desequilíbrios articulares em função do desconhecimento dos níveis de estresses funcionais e das consequentes adaptações musculares provocados entre os exercícios monoarticulares e multiarticulares.

Devido à carência de estudos, e instrumentos metodológicos para avaliar o desempenho muscular em ações sinérgicas, principalmente para músculos agonistas e em exercícios resistidos, o objetivo deste estudo foi verificar a magnitude da realização do tríceps na polia no estresse funcional no supino reto.

\section{METODOLOGIA}

\subsection{Amostra}

A amostra foi composta por 10 indivíduos, do sexo masculino, com experiência não inferior a 12 meses no treinamento resistido, idade média de 24,9 $\pm 3,17$ anos, massa corporal média de 78,9 $\pm 11,48 \mathrm{~kg}$ e estatura média de $1,79 \pm 0,05 \mathrm{~cm}$. Para participarem, os voluntários deveriam responder negativamente o questionário PAR-Q, não fazer uso de substâncias ergogênicas, praticar treinamento de força com frequência mínima de 3 (três) dias na semana e não apresentar problemas articulares ou musculares que pudessem interferir no desempenho. Foram excluídos os voluntários que não se apresentaram para os testes, não relataram estar alimentados normalmente às suas 
rotinas; não relataram uma boa qualidade na noite de sono anterior (relativa ao comportamento individual normal); e não se empenharam na execução das tarefas pré-determinadas. O protocolo de pesquisa (074270/2019) foi aprovado pelo Comitê de Ética e Pesquisa dos Institutos Superiores de Ensino do CENSA.

\subsection{Procedimentos}

Os indivíduos realizaram 3 visitas à academia. O primeiro dia consistiu na aplicação do teste de 10 RMs no tríceps na polia alta. Em sequência, 5 minutos de descanso para realizar o teste de 10 RMs no supino reto. Para obter os $10 \mathrm{RMs}$, cada indivíduo teve 5 tentativas máximas para que o teste fosse validado. Os 10 RMs foram avaliados em dois dias com intervalo de 48 horas entre os testes. No segundo dia, cada indivíduo realizou um aquecimento muscular específico no supino reto utilizando 2 séries de $40 \%$ da carga obtida no teste de 10RMs. Após as séries de aquecimento, os indivíduos realizaram três séries com $85 \%$ da carga dos 10 RMs do Supino Reto até a exaustão voluntária máxima (isto é, falha muscular concêntrica) utilizando uma carga constante em todas séries. $\mathrm{O}$ intervalo entre as séries foi de 1 minuto e foram contabilizadas as repetições máximas realizadas.No terceiro dia de testes após as séries de aquecimento, os indivíduos realizaram uma série de 10 RMs no tríceps na polia com a carga que foi verificada para 10 RMs. Imediatamente após a série no tríceps na polia, os indivíduos realizaram o Supino Reto com $85 \%$ da carga dos mesmos 10 RMs encontrados antriormente. Todos realizaram 3 séries até a exaustão voluntária máxima utilizando uma carga constante com um intervalo de 1 minuto entre as séries. As sessões de exercício foram realizadas nos mesmos dias e horários da semana. Foi estabelecido um intervalo de 48 horas entres os dias das sessões de testes. Todos os procedimentos estão descritos na figura 1.

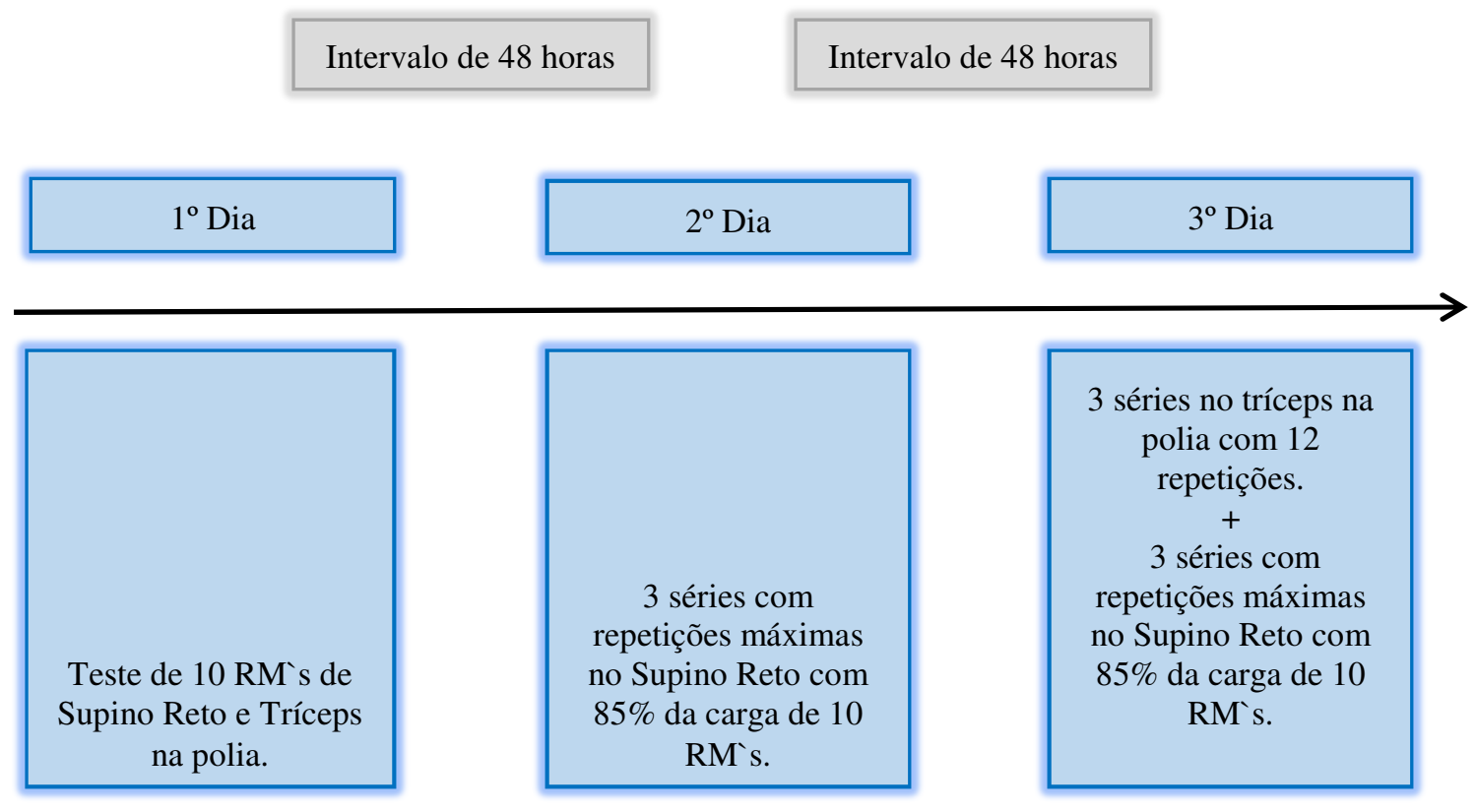

Figura 1- Desenho Experimental

\subsection{Análise Estatística}

Foi utilizada a estatística descritiva média \pm desvios padrão (Média $\pm D P$ ). O teste ShapiroWilk foi utilizado para verificar a normalidade dos dados. Para a análise inferencial foi utilizada a análise de variância (ANOVA-Two-way) com o teste de post hoc de Tukey's para os parâmetros de 
repetições e volume total. Foi considerado significativo o valor de $p<0,05$. As análises estatísticas foram realizadas utilizando o software SPSS 16.0 (SPSS Inc., Chicago, IL, EUA).

\section{RESULTADOS}

Na figura 2 é mostrada o número de repetições máximas nas 3 séries, nos 2 ensaios experimentais ( $\mathrm{SR}$ e $\mathrm{TR}+\mathrm{SR}$ ). Observou-se uma redução no número de repetições realizadas na $1^{\mathrm{a}}$ série no ensaio $\mathrm{TR}+\mathrm{SR}$ comparado ao $\mathrm{SR}(\mathrm{p}<0,05)$. Não houve redução significativa no número de repetições realizadas na $2^{\mathrm{a}}$ e $3^{\mathrm{a}}$ séries comparada ao $\mathrm{SR}(\mathrm{p}>0,05)$.

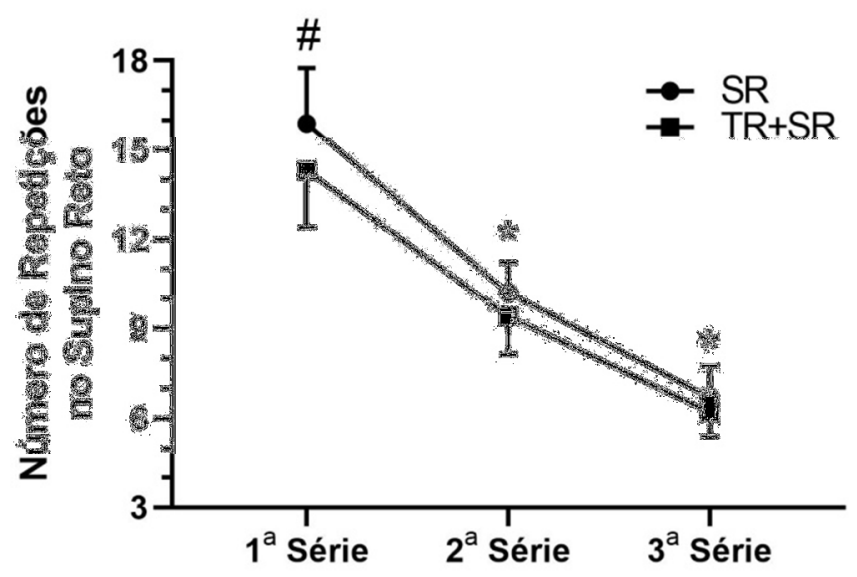

Figura 2 - Número de repetições máximas realizadas no supino reto nos 2 ensaios experimentais (Média $\pm D P$ ). SR - Supino Reto; TR+SR - Tríceps na Polia + Supino Reto. *diferente da $1^{\text {a }}$ série. \#diferente entre SR e TR+SR.

Na figura 3 é mostrada o volume total no supino reto nas 3 séries alcançadas, nos 2 ensaios experimentais (SR e TR+SR). Observou-se uma diminuição no volume total na $1^{a}$ série no ensaio $\mathrm{TR}+\mathrm{SR}$ comparado ao $\mathrm{SR}(\mathrm{p}<0,05)$. Não houve redução significativa no volume total na $2^{\mathrm{a}}$ e $3^{\mathrm{a}}$ séries comparada ao SR ( $\mathrm{p}>0,05)$.

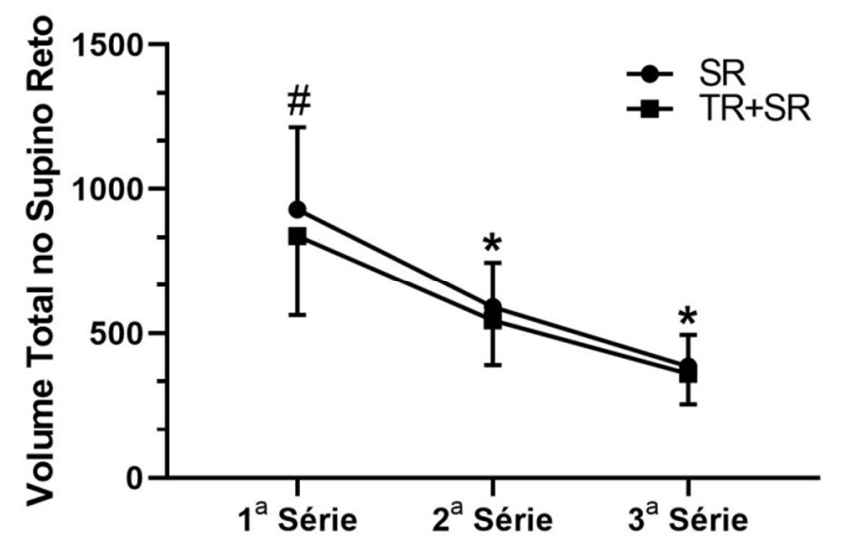

Figura 3 - Volume total no supino reto nos 2 ensaios experimentais (Média $\pm D P$ ). SR - Supino Reto; TR+SR - Tríceps na Polia + Supino Reto. *diferente da $1^{\text {a }}$ série. \#diferente entre SR e $\mathrm{TR}+\mathrm{SR}$. 


\section{DISCUSSÃO}

Analisando os achados nas duas perspectivas abordadas na presente pesquisa, foi possível verificar apenas na $1^{a}$ série do exercício, uma redução nas repetições realizadas no supino reto após a realização do exercício tríceps na polia.

De acordo com Simão et al (2007), a ordem dos exercícios referem-se à sequência em que eles são realizados em uma sessão de treinamento, a qual parece influenciar diretamente o número de repetições e consequentemente o volume total de trabalho. Shimano et al (2006) observaram que o número de repetições realizadas com uma mesma carga relativa está relacionado com o tamanho do grupo muscular envolvido no exercício. Sforzo \& Touey (1996) relataram que a ordem de realização dos exercícios poderia afetar de maneira distinta o número de repetições realizadas em grupos musculares de tamanhos diferentes. No presente estudo, utilizando o estresse funcional de um exercício monoarticular (tríceps na polia), seguido da realização de um multiarticular (supino reto), observou-se uma redução significativa no número de repetições na $1^{\text {a }}$ série no total de 3 séries. Acredita-se que o número de repetições realizadas para o múculo TB antes da realização do supino contribuiu para uma redução no número de repetições e o volume total obeservados na primeira série do exercício de supino, quando o músculo peitoral não se encontrava na condição momentânea de fadiga.

$\mathrm{Na}$ segunda e terceira séries, não foi observada redução significativa no número de repetições realizadas no supino, utilizando previamente a realização de um exercício monoarticular (tríceps na polia) comparada à realização do exercício de supino $(\mathrm{p}<0.05)$. Provavelmente, esta diferença foi provocada pelas questões metabólicas envolvidas na atividade muscular do múculo peitoral, somadas às questões neurais (ESCAMILLA et al., 2009). Estes dados sugerem que, com esta combinação de exercícios, houve uma depressão das capacidades funcionais do sistema nervoso central e das reservas de glicogênio intramusculares (GENTIL et al., 2007). Simão et al (2005) afirmam como hipótese inicial, que a exaustão de um músculo com ação sinérgica, promoveria redução da capacidade funcional durante a realização de exercícios multiarticulares. Dessa forma, pode-se inferir que quando há instalação da fadiga do músculo peitoral em um determinado número de séries executadas, o estresse funcional do TB pode não interferir na performance do exercício de supino. Esses achados devem ser analisados com cuidado, pois a presente pesquisa apresenta algumas limitações, como a ausência de controle da velocidade de execução e a amplitude do movimento.

\section{CONCLUSÕES}

Conclui-se que a execução do exercício de tríceps na polia precedido ao exercício de supino resultou no estresse funcional somente na $1^{\mathrm{a}}$ série e, que a presença da fadiga do músculo peitoral apresentada nas séries subsequentes não impactou no aspecto funcional na realização do exercício de supino. Intervenções em relação à ordem de exercícios podem auxiliar os profissionais de Educação Física a adotarem estratégias na manipulação de variáveis do treinamento.

\section{REFERÊNCIAS}

AMERICAN COLLEGE OF SPORTS MEDICINE (ACSM). American College of Sports Medicine position stand. Progression models in resistance training for healthy adults. Medicine e 
Science in Sports e Exercise, v. 41, n. 3, p. 687-708, $2009 . \quad$ DOI: 10.1249/MSS.0b013e3181915670

ASMUSSEN, E. Muscle Fatigue. Medicine and Science in Sports, v. 11, n. 4, p. 313-321, 1979.

AUGUSTSSON, J.; THOMEÉ, R.; HORNSTEDT, P.; LINDPLOM, J.; KARLSSON, J.; GRIMBY, A.G. Effect of pre-exhaustion exercise on lower-extremity muscle activation during a leg press exercise. The Journal of Strength and Conditioning Research, v. 17, n. 2, p. 411-416, 2003.

ESCAMILLA, R.F.; FLEISIG, G.S.; ZHENG, N.; LANDER, J.E.; BARRENTINE, S.W.; ANDREWS, J.R.; BERGEMANN, B.W.; MOORMAN, C.T. Effects of technique variations on knee biomechanics during the squat and leg press. Medicine and Science in Sports and Exercise, v. 33, n. 9, p. 1552-1566, 2001.

ESCAMILLA, R.F.; ZHENG, N.; MACLEOD, T.D.; EDWARDS, W.B.; IMAMURA, R.; HRELJAC, A.; FLEISIG, G;S.; WILK, K.E.; MOORMAN III, C.T.; ANDREWS, J.R. Patellofemoral joint force and stress during the squat and one-leg squat. Medicine and Science in Sports and Exercise, v. 41, n. 4, p. 879-888, 2009. DOI: 10.1249/MSS.0b013e31818e7ead

GENTIL, P.; OLIVEIRA, E.; JUNIOR V.A.R.; CARMO, J.; BOTTARO, M. Effects of exercise order on upper-body muscle activation and exercise performance. The Journal of Strength and Conditioning Research, v. 21, n. 4, p. 1082-1086, 2007.

GUYTON, A.C.; HALL, J.E. Textbook of medical physiology. $11^{\mathrm{a}}$ Ed. Philadelphia, Pennsylvania: Elsevier Saunders, 2006.

HÄKKINEN, K.; PAKARINEN, A.; KRAEMER, W.J.; HÄKKINEN, A.; VALKEINEN, H.; ALEN, M. Selective muscle hypertrophy, changes in EMG and force, and serum hormones during strength training in older women. Journal of Applied Physiology, v. 91, n. 2, p. 569-580, 2001. DOI: 10.1152/jappl.2001.91.2.569

JORGE, F.S.; TINOCO, M.L.A.; TAVARES, T.G.DE O.; MORALES, A.P. Influência da bandagem neuromuscular no desempenho do salto vertical. Perspectivas Online: Biológicas \& Saúde, v. 2, n. 7, p. 62-75, 2012. DOI: 10.25242/8868272012198

KRAEMER, W. J., MAZZETTI, S. A., NINDL, B. C., GOTSHALK, L. A., VOLEK, J. S., BUSH, J. A., MARX, J. O., DOHI, K., GÓMEZ, A. L., MILES, M., FLECK, S. J. Effect of resistance training on women's strength/power and occupational performances. Medicine \& Science in Sports \& Exercise, v. 33, n. 6, p. 1011-1025, 2001.

KRAEMER, W.J. et al. Effect of resistance training on women's strength/power and occupational performances. Medicine \& Science in Sports \& Exercise, v. 33, n. 6, p. 1011-1025, 2001.

PAOLI, A.; MARCOLIN, G.; PETRONE, N. The effect of stance width on the electromyographical activity of eight superficial thigh muscles during back squat with different bar loads. The Journal of Strength and Conditioning Research, v. 23, n. 1, p. 246-250, 2009. DOI: 10.1519/JSC.0b013e3181876811

ORNELAS, C.DOS S.; DE ALMEIDA, M.W.S.; DE OLIVEIRA, M.B.C.; DA SILVA, V.F.; E SILVA, A.P.R.DE S.; CALOMENI, M.R. O efeito da prática de musculação associada ao 
treinamento mental e estimulação cerebral no desenvolvimento do bíceps braquial. Perspectivas Online: Biológicas \& Saúde, v. 7, n. 23, p. 1-8, 2017. DOI: 10.25242/886872320171109

SFORZO, G.; TOUEY, P. Manipulating exercise order affects muscular performance during a resistance exercise training session. The Journal of Strength and Conditioning Research, v. 10, n. 1, p. 20-24, 1996.

SHIMANO, T.; KRAEMER, W. J.; SPIERING, B.A.; VOLEK, J.S.; HATFIELD, D.L.; SILVESTRE, R.; VINGREN, J.L.; FRAGALA, M.S.; MARESH, C.M.; FLECK, S.J.; NEWTON, R.U.; SPREUWENBERG, L.P.B.; HAKKINEN, K. Relationship between the number of repetitions and selected percentages of one repetition maximum in free weight exercises in trained and untrained men. The Journal of Strength and Conditioning Research, v. 20, n 4, p. 819-823, 2006. DOI: $10.1519 / \mathrm{R}-18195.1$

SIMÃO, R.; FARINATTI, P. T. V.; POLITO, M. D.; MAIOR, A. S.; FLECK, S. J. Influence of exercise order on the number of repetitions performed and perceived exertion during resistive exercises. The Journal of Strength and Conditioning Research, v. 19, n. 1, p. 152-156, 2005. DOI: $10.1519 / 1533-4287(2005) 19<152:$ IOEOOT $>2.0 . C O ; 2$

SIMÃO, R.; FARINATTI, P. T.; POLITO, M. D.; VIVEIROS, L.; FLECK, S.J. Influence of exercise order on the number of repetitions performed and perceived exertion during resistance exercise in women. The Journal of Strength and Conditioning Research, v. 21, n. 1, p. 23-28, 2007. DOI: 10.1519/00124278-200702000-00005

TESCH, P.A. Target bodybuilding. Champaingn: Human kinetics, 1999. 